\title{
Lutein and Zeaxanthin Influence Brain Function in Older Adults: A Randomized Controlled Trial
}

\author{
Cutter A. Lindbergh, ${ }^{1}$ Lisa M. Renzi-Hammond, ${ }^{1}$ Billy R. Hammond, ${ }^{1}$ Douglas P. Terry, ${ }^{1}$ Catherine M. Mewborn, ${ }^{1}$ \\ Antonio N. Puente, ${ }^{1}$ AND L. Stephen Miller ${ }^{1,2}$ \\ ${ }^{1}$ Department of Psychology, University of Georgia, Athens, Georgia \\ ${ }^{2}$ Bio-Imaging Research Center, Paul D. Coverdell Center, University of Georgia, Athens, Georgia \\ (Received December 17, 2016; Final Revision May 18, 2017; Accepted May 22, 2017; First Published Online July 11, 2017)
}

\begin{abstract}
Objectives: The present study constitutes the first randomized controlled trial to investigate the relation of lutein (L) and zeaxanthin ( $\mathrm{Z}$ ) to brain function using functional magnetic resonance imaging (fMRI). It was hypothesized that $\mathrm{L}$ and $\mathrm{Z}$ supplementation in older adults would enhance neural efficiency (i.e., reduce activation) and cognitive performance on a verbal learning task relative to placebo. Methods: A total of 44 community-dwelling older adults (mean age $=72$ years) were randomly assigned to receive either placebo or $\mathrm{L}+\mathrm{Z}$ supplementation $(12 \mathrm{mg} /$ daily) for 1 year. Neurocognitive performance was assessed at baseline and post-intervention on an fMRI-adapted task involving learning and recalling word pairs. Imaging contrasts of blood-oxygen-level-dependent (BOLD) signal were created by subtracting active control trials from learning and recall trials. A flexible factorial model was employed to investigate the expected group (placebo $v s$. supplement) by time (baseline $v s$. post-intervention) interaction in pre-specified regions-of-interest. Results: $\mathrm{L}$ and $\mathrm{Z}$ appeared to buffer cognitive decline on the verbal learning task (Cohen's $d=.84$ ). Significant interactions during learning were observed in left dorsolateral prefrontal cortex and anterior cingulate cortex $(p<.05$, family-wise-error corrected). However, these effects were in the direction of increased rather than decreased BOLD signal. Although the omnibus interaction was not significant during recall, within-group contrasts revealed significant increases in left prefrontal activation in the supplement group only. Conclusions: $\mathrm{L}$ and $\mathrm{Z}$ supplementation appears to benefit neurocognitive function by enhancing cerebral perfusion, even if consumed for a discrete period of time in late life. (JINS, 2018,
\end{abstract}

$24,77-90)$

Keywords: Aging, Cognition, Diet, Food, Magnetic resonance imaging, Carotenoids

\section{INTRODUCTION}

The xanthophyll lutein (L) and its isomer zeaxanthin $(\mathrm{Z})$ are among over 600 organic pigments within the carotenoid family (Britton, Liaaen-Jensen, \& Pfander, 2004; Khachik, Beecher, Goli, \& Lusby, 1991). L and Z are not produced endogenously and thus must be consumed through green vegetables, colored fruits, and other aspects of diet (Hammond et al., 1997; Malinow, Feeney-Burns, Peterson, Klein, \& Neuringer, 1980). They traverse the blood-retina barrier to accumulate in several regions of the eye (Bernstein et al., 2001) but are taken up most selectively in the central region of the retina, referred to as the macula (Bone, Landrum, \& Tarsis, 1985). The importance of $\mathrm{L}$ and $\mathrm{Z}$ to eye health is well-established, particularly in protecting

Correspondence and reprint requests to: L. Stephen Miller, Department of Psychology, Psychology Building, University of Georgia, Athens, GA 30602-3013. E-mail: 1smiller@uga.edu against age-related macular degeneration (Ma et al., 2012; SanGiovanni \& Neuringer, 2012).

$\mathrm{L}$ and $\mathrm{Z}$ also accumulate preferentially in human brain tissue (Craft, Haitema, Garnett, Fitch, \& Dorey, 2004; Johnson et al., 2013). Together with other xanthophylls (e.g., B-cryptoxanthin), $\mathrm{L}$ and $\mathrm{Z}$ account for two-thirds or more of total brain carotenoid concentrations (Craft et al., 2004).

Most studies determine $\mathrm{L}$ and $\mathrm{Z}$ levels by measuring blood serum concentrations or by evaluating the optical density of the macular pigment layer (MPOD; Hammond, Wooten, \& Smollon, 2005). Serum levels tend to be influenced by recent food consumption while MPOD reflects longer-term dietary habits (Hammond et al., 1997). MPOD correlates with postmortem brain tissue concentrations in primates and humans and is considered the most reliable proxy for neural levels (Hammond et al., 2005; Vishwanathan, Neuringer, Snodderly, Schalch, \& Johnson, 2013; Vishwanathan, Schalch, \& Johnson, 2016). 
Human and animal studies have shown a relationship between $\mathrm{L}$ and $\mathrm{Z}$ dietary intake and MPOD. Rhesus monkeys raised on xanthophyll-free diets exhibit absent or severely depleted macular pigment (Malinow et al., 1980). In older women $(N=1698)$, self-reported dietary intake of $\mathrm{L}$ and $\mathrm{Z}$ positively correlated with MPOD, even after adjusting for medical and lifestyle factors (Mares et al., 2006). Adults who augmented their diets with 60 grams of spinach daily for 15 weeks showed a $19 \%$ increase in MPOD on average (Hammond et al., 1997). L and Z supplements also significantly increase MPOD in both young and older adults (e.g., Landrum et al., 1997; Murray et al., 2013; Stringham \& Hammond, 2008; Weigert et al., 2011). For example, Stringham and Hammond (2008) found 6 months of L and Z supplementation (12 $\mathrm{mg} /$ daily) to significantly increase MPOD in healthy adults (Cohen's $d=0.97$ ) and improve visual performance. Several other studies have similarly reported improvements in visual function resulting from supplementation (e.g., Kvansakul et al., 2006; Ma et al., 2009; Yagi et al., 2009), suggesting functional benefits of enhancing MPOD.

An emerging literature also suggests a relation of $\mathrm{L}$ and $\mathrm{Z}$ dietary intake to cognition in older adults (for reviews, see: Erdman et al., 2015; Johnson, 2012, 2014; Maci, Fonseca, \& Zhu, 2016). For example, a carotenoid-rich dietary pattern in late middle age was associated with better episodic memory, verbal fluency, working memory, and executive functioning 13 years into the future (Kesse-Guyot et al., 2014). Similarly, greater intake of vegetables containing $\mathrm{L}$ and $\mathrm{Z}$ has been longitudinally associated with slower rates of cognitive decline in older adults (Kang, Ascherio, Grodstein, 2005; Lee, Kim, \& Back, 2009; Morris, Evans, Tangney, Bienias, \& Wilson, 2006). MPOD and serum $\mathrm{L}$ and/or $\mathrm{Z}$ concentrations have been found to positively correlate with a range of cognitive functions in older adults (Akbaraly, Faure, Gourlet, Favier, \& Berr, 2007; Feeney et al., 2013; Johnson et al., 2013; Kelly et al., 2015; Renzi, Dengler, Puente, Miller, \& Hammond, 2014; Renzi, Iannacocone, Johnson, \& Kritchevsky, 2008; Vishwanathan et al., 2014). Greater serum L is associated with reduced risk for dementia and Alzheimer's disease mortality (Feart et al., 2015; Min \& Min, 2014). In addition, Alzheimer's disease patients have lower MPOD and serum $\mathrm{L}$ and $\mathrm{Z}$ relative to age-matched controls (Nolan et al., 2014).

Several reviews have called for randomized controlled trials (RCTs) to determine whether low carotenoid levels contribute to cognitive loss or perhaps represent a consequence, either through neuropathological processes or poor nutritional decisions (Erdman et al., 2015; Johnson, 2012, 2014). To the authors' knowledge, only one such RCT exists (Johnson et al., 2008) in a sample of older women randomly assigned to receive 4 months of daily DHA (a polyunsaturated fatty acid; $n=14)$, L $(n=11)$, combined supplementation $(n=14)$, or placebo $(n=14)$. The group receiving $\mathrm{L}$ showed significant gains in verbal fluency relative to placebo at 4 months $(d=0.61)$. The combined $(\mathrm{DHA}+\mathrm{L})$ group evidenced improvements in verbal fluency $(d=0.90)$, learning $(d=0.70)$, and memory $(d=0.58)$ at 4 months.

The means by which $\mathrm{L}$ and $\mathrm{Z}$ influence brain function is an understudied area, but several mechanisms have been proposed (Erdman et al., 2015; Zamroziewicz \& Barbey, 2016). $\mathrm{L}$ and $\mathrm{Z}$ have strong antioxidant and anti-inflammatory properties, which may counter the deleterious effects of agerelated oxidative stress and neuroinflammation (Bokov, Chaudhuri, \& Richardson, 2004; Butterfield, Bader Lange, \& Sultana, 2010; Heneka et al., 2015; Rosano, Marsland, \& Gianaros, 2012). L and $\mathrm{Z}$ may also benefit neurocognitive function through their positive effects on cell membrane fluidity, permeability, stability, thickness, and ion exchange (Erdman et al., 2015; Krinsky, Mayne, \& Sies, 2004; Widomska \& Subczynski, 2014). Relatedly, carotenoids such as $\mathrm{L}$ and $\mathrm{Z}$ may improve cellular communication by altering gene expression (e.g., connexin 43; Bertram, 1999) and enhancing inter-neuronal signaling at gap junctions (Stahl \& Sies, 2001).

The present study was a 12-month RCT that sought to expand the research base on neural mechanisms underlying the relation of $\mathrm{L}$ and $\mathrm{Z}$ to cognition in community-dwelling older adults using functional magnetic resonance imaging (fMRI). Cross-sectional results from the study sample at baseline (Lindbergh et al., 2016) revealed a negative relationship of pre-intervention $\mathrm{L}$ and $\mathrm{Z}$ levels (measured via serum and MPOD) to blood-oxygen-level-dependent (BOLD) signal in several brain regions during an fMRIadapted verbal learning task. Lindbergh et al. (2016) interpreted the observed results to reflect a potential role of $\mathrm{L}$ and $\mathrm{Z}$ in promoting neural efficiency (the neural efficiency hypothesis; Renzi \& Hammond, 2010) during cognitive performance, particularly in brain regions with established risk for age-related degeneration. This interpretation was based in part on the revised Scaffolding Theory of Aging and Cognition (STAC-r), which emphasizes "life-course" variables, including nutrition, in shaping the structure and function of the aging brain (Reuter-Lorenz \& Park, 2014). More specifically, Lindbergh et al. (2016) speculated that $\mathrm{L}$ and $\mathrm{Z}$ buffer age-related neuropathological processes and thus reduce the associated need for compensatory neural recruitment in response to cognitive challenge (Park \& Reuter-Lorenz, 2009; Reuter-Lorenz \& Park, 2014). Although $\mathrm{L}$ and $\mathrm{Z}$ are not specifically mentioned in the STAC-r, this would be consistent with the more general construct of "neural resource enrichment" discussed by Reuter-Lorenz and Park (2014, p. 361), which encompasses various nutritional and lifestyle factors. As with most research on this topic, however, Lindbergh et al.'s (2016) conclusions were limited by the cross-sectional nature of the analyses.

The RCT design of the current investigation addressed limitations of cross-sectional analyses and permitted evaluation of $\mathrm{L}$ and $\mathrm{Z}$ 's effects on the aging brain relative to placebo. An fMRI-adapted verbal learning and memory paradigm was employed in which participants were asked to learn and recall word pairs. Within the framework of the 
STAC-r (Reuter-Lorenz \& Park, 2014), L and Z were expected to function as "neural resource enrichment," ultimately countering the effects of neurophysiological decline and reducing the need for compensatory scaffolding. Accordingly, it was expected that older adults receiving $\mathrm{L}$ and $\mathrm{Z}$ supplementation would exhibit increased neurobiological efficiency as evidenced by reduced neural activity (i.e., BOLD signal) required to meet task demands relative to controls. Based on regions-of-interest (ROIs) identified through prior literature (e.g., Bookheimer et al., 2000, 2013; Cabeza \& Nyberg, 2000; Clément \& Belleville, 2009), the anticipated effects during verbal learning were expected to predominate in medial temporal lobe, supramarginal and angular gyri, precuneus, dorsolateral and ventrolateral prefrontal cortex, anterior and posterior cingulate gyrus, Broca's area, cerebellum, and premotor areas. A similar network of brain regions was expected to show effects during verbal recall with the addition of anterior prefrontal cortex and medial parieto-occipital regions (retrosplenial cortex and cuneus) given somewhat different processes involved in retrieval versus encoding (Cabeza \& Nyberg, 2000).

A secondary aim was to replicate and extend previous findings showing a positive relation of $\mathrm{L}$ and $\mathrm{Z}$ to cognition in older adults. Consistent with the bulk of the available literature, it was hypothesized that L and Z supplementation would benefit performance on the fMRI-adapted verbal learning task in terms of number of words successfully recalled relative to placebo.

\section{METHOD}

\section{Participants}

75 community-dwelling older adults (64-86 years of age) were recruited via newspaper advertisements, flyers, and electronic media (e.g., listservs) as part of a larger intervention study. Exclusionary criteria included left-handedness, traumatic brain injury, macular degeneration, gastric conditions that may interfere with supplement absorption, corrected visual acuity worse than 20:40, MRI incompatibility, Geriatric Depression Scale (GDS) total score $>19$, or neurological disorder. Of the 75 participants eligible for inclusion following an initial phone screening, more thorough medical history review revealed that seven participants were incompatible with the MRI environment. Three more participants became uncomfortable within the scanner and requested to stop due to claustrophobia $(n=2)$ or sensitivity to noise $(n=1)$. An additional participant was unable to remain awake. Sixteen volunteers elected not to complete the study for various reasons, and behavioral data for one individual were lost due to technical malfunction. Three volunteers were obviously unable to grasp the fMRI task, yielding a final sample size of 44 (experimental $n=30$; control $n=14$ ) for analyses.

\section{Procedures}

A single-site, double-blind, RCT design was employed. Eligible participants were assigned using a 2:1 experimental to control group ratio to receive either a supplement containing $\mathrm{L}(10 \mathrm{mg})$ and $\mathrm{Z}(2 \mathrm{mg})$ or a physiologically inert placebo of identical appearance provided by DSM Nutritional Products (Basel, Switzerland). Randomization was performed by generating a set of numerical codes corresponding to either the active supplement or the placebo. These codes were placed in an opaque envelope and drawn for each participant by the study coordinator, who did not have any data collection responsibilities. Participants were instructed to consume one pill per day with a meal for 1 year. As noted previously, baseline (cross-sectional) fMRI findings from this study evaluating the relation of naturally circulating $\mathrm{L}$ and $\mathrm{Z}$ levels to neural activity, prior to supplementation, have been published elsewhere (Lindbergh et al., 2016). Lindbergh et al. (2016) used a largely overlapping, although not completely identical, subset of the sample in final analyses due to somewhat different variables of interest (see Lindbergh et al., 2016).

The RCT entailed eight laboratory visits in addition to a physical examination with a health professional to verify appropriate health for the study (see Table 1). Eligible participants completed three baseline sessions occurring within a 2-week period, including vision testing, cognitive testing, measurement of $\mathrm{L}$ and $\mathrm{Z}$ concentrations in macular pigment, and acquisition of neuroimaging data. Bi-monthly contact

Table 1. Study timeline

\begin{tabular}{|c|c|c|c|c|c|c|c|c|c|}
\hline & \multirow{2}{*}{$\frac{\text { Pre-screen }}{\text { Health center }}$} & \multicolumn{3}{|c|}{ Baseline } & \multirow{2}{*}{$\frac{\text { 4-Mos }}{\text { Visit } 4}$} & \multirow{2}{*}{$\frac{8 \text {-Mos }}{\text { Visit } 5}$} & \multicolumn{3}{|c|}{ Post-intervention } \\
\hline & & Visit 1 & Visit 2 & Visit 3 & & & Visit 6 & Visit 7 & Visit 8 \\
\hline Physical exam & $\checkmark$ & & & & & & & & \\
\hline Vision testing & & & $\checkmark$ & & $\checkmark$ & $\checkmark$ & & $\checkmark$ & \\
\hline Blood draw & & & $\checkmark$ & & $\checkmark$ & $\checkmark$ & & $\checkmark$ & \\
\hline Cog. testing & & $\checkmark$ & & & $\checkmark$ & $\checkmark$ & $\checkmark$ & & \\
\hline Neuroimaging & & & & $\checkmark$ & & & & & $\checkmark$ \\
\hline Pill counts & & & & & $\checkmark$ & $\checkmark$ & $\checkmark$ & & \\
\hline
\end{tabular}

Note. Cog. testing = cognitive testing, which included the Geriatric Depression Scale and Wechsler Test of Adult Reading at Visit 1 ; Mos $=$ months. Baseline visits 1,2 , and 3 occurred within a 2 -week window, as did post-intervention visits 6,7 , and 8 . The post-intervention visits occurred at 12 months. Vision testing included measurement of macular pigment optical density (MPOD). 
assessed compliance, adverse events, and any health changes that could render participants ineligible for participation. Pill counts were conducted at 4 month, 8 month, and 12 month visits and participants were only provided with enough pills to last until their next visit. The 12 month (post-intervention) visit was completed across three sessions that mirrored the baseline visit.

Participants were compensated with $\$ 300$ distributed across four time points (i.e., baseline, 4 months, 8 months, and 12 months). If participants required transportation to experimental sessions, $\$ 20$ was provided to the collateral driver at each time point.

The study was approved by the University of Georgia Institutional Review Board and the tenets of the Declaration of Helsinki were closely followed by all study personnel.

\section{Measures}

\section{Wechsler Test of Adult Reading}

Premorbid intellectual functioning was estimated using the Wechsler Test of Adult Reading (WTAR), which involves reading a list of 50 words with atypical grapheme to phoneme relationships (Wechsler, 2001). Full-scale intelligence quotient estimates were calculated using an algorithmic combination of WTAR performance (i.e., number of words correctly pronounced) and demographic (i.e., age, education, race, sex, and geographic region) variables (Wechsler, 2001).

\section{GDS}

The GDS was used to screen for significant depressive symptomatology (Yesavage et al., 1983). The GDS is a self-report questionnaire consisting of 30 items with yes/no response options, yielding a total score ranging from 0 to 30 (0-9 = normal; $10-19=$ mild; $20-30=$ severe $)$.

\section{MPOD}

$\mathrm{L}$ and $\mathrm{Z}$ accumulate preferentially in the central area of the retina, referred to as the macula (Bone et al., 1985). Pigment within the human macula consists of $\mathrm{L}$ and $\mathrm{Z}$ embedded in retinal tissue (Bone et al., 1985). The density of the macular pigment layer (MPOD) is frequently used to index $\mathrm{L}$ and $\mathrm{Z}$ levels within the central nervous system (e.g., Vishwanathan et al., 2016) and can be measured noninvasively using customized heterochromatic flicker photometry (cHFP), a widely validated technique (Stringham et al, 2008). The reader is referred to our published baseline findings for a detailed description of the cHFP procedure (Lindbergh et al., 2016), although it is important to note that Lindbergh et al. (2016) used MPOD as a predictor (i.e., independent variable) in the fMRI analyses. By contrast, in the present analyses, MPOD data are presented only as a validity check on our intervention to verify that our supplementation effectively increased $\mathrm{L}$ and $\mathrm{Z}$ status over the course of the study. Briefly, cHFP involved presenting a 1-deg visual stimulus consisting of two narrow-band light sources, peaking at $460 \mathrm{~nm}$ and $570 \mathrm{~nm}$, using a macular densitometer (Macular Metrics; Rehoboth, MA). After determining customized flicker sensitivities, the lower (i.e., $460 \mathrm{~nm}$ ) waveband radiance was manipulated relative to the $570 \mathrm{~nm}$ waveband to measure the point at which flickering could no longer be perceived. This sequence was conducted again with a $2^{\circ}$ target and fixation point at $7^{\circ}$ nasally to provide a parafoveal reference measurement where MPOD approaches zero. The two loci were then contrasted for an MPOD measurement at 30 minutes of retinal eccentricity.

\section{Neuroimaging}

\section{FMRI task}

Participants engaged in a verbal learning task, conceptually derived from the Wechsler memory scale paired associates learning test (Wechsler, 2009), similar to previously published fMRI paradigms (e.g., Bookheimer et al., 2000, 2013; Braskie, Small, \& Bookheimer, 2009), and described in detail elsewhere (Lindbergh et al., 2016). The basic goal of the task was to learn unrelated word pairs (e.g., "UP" and "FOOT"). E-Prime software (version 1.2, Psychology Software Tools, Inc., Pittsburgh, PA) was used to program and present the task in conjunction with MRI compatible goggles (Resonance Technology Inc., Northridge, CA). Participants provided responses using right and left index finger buttons on Cedrus Lumina LU400 MRI compatible response pads (Cedrus, San Pedro, CA).

The task entailed 10 each of learning blocks, control blocks, retrieval blocks, and baseline fixation blocks (see Figure 1). During learning blocks, the first word of each pair was displayed in isolation on the left side of the screen (1 s) followed by presentation of the second word on the right side, such that both words could be viewed side by side ( $2 \mathrm{~s})$. There were 10 word pairs total with 5 pairs presented during each encoding block. During retrieval trials, participants were presented with the first word in each pair (3s) and asked to mentally recall the second word to prevent head motion, consistent with procedures used in analogous fMRI-adapted verbal learning tasks (e.g., Bookheimer et al., 2000, 2013). Participants were instructed to make a right index finger press if they successfully retrieved the second word or a left index finger press to indicate unsuccessful retrieval. A control task interspersed learning and retrieval blocks, which mimicked the learning block except that "XXXXX" and "YYYYY" were presented in place of word pairs. Immediately post scan, participants engaged in cued recall of the "second word" in each pair (maximum score: 10) to help assess task engagement within the scanner.

\section{MRI acquisition}

Scans were acquired on a General Electric (GE; Waukesha, WI) $3 \mathrm{~T}$ Signa HDx MRI system. A high-resolution threedimensional T1-weighted fast spoiled gradient recall echo sequence was used to collect structural scans (repetition time $[\mathrm{TR}]=7.5 \mathrm{~ms}$; echo time $[\mathrm{TE}]=<5 \mathrm{~ms}$; field of view 


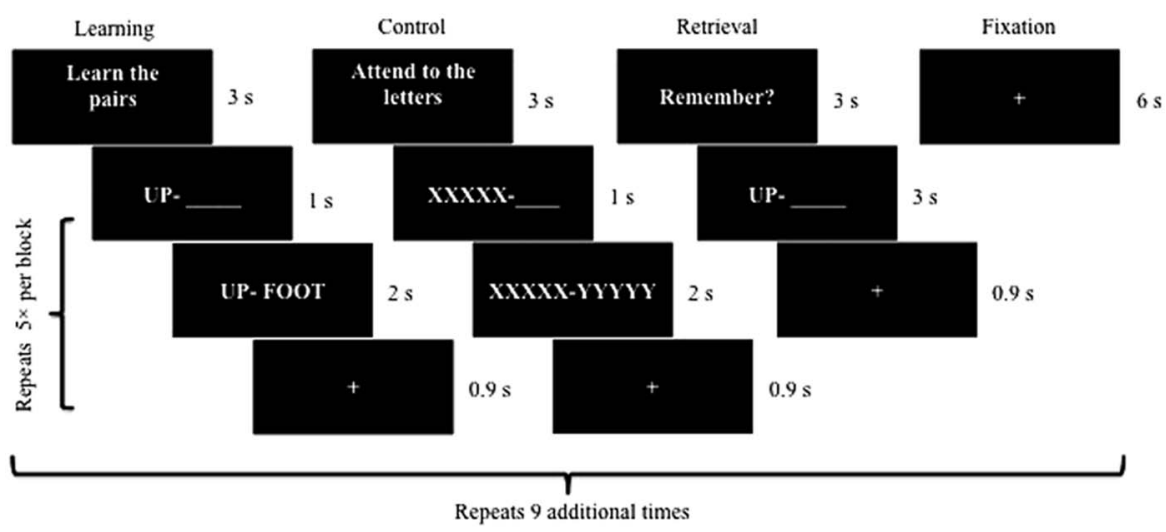

Fig. 1. Visual schematic of the fMRI-adapted verbal learning task. Each block (i.e., learning, control, retrieval, and fixation) was presented 10 times. Five different word pairs were included within every learning block followed by five XXXXX - YYYYY pairings within every control block. Similarly, participants attempted to recall the second word of five different word pairs during each retrieval block.

$[\mathrm{FOV}]=256 \times 256 \mathrm{~mm}$ matrix; flip angle $=20^{\circ}$; slice thickness $=1.2 \mathrm{~mm} ; 154$ axial slices) with a total acquisition time of 6 min and $20 \mathrm{~s}$. This protocol collected 176 images, providing coverage from the brainstem to the top of the head.

Functional scans were aligned to each participant's anterior commissure-posterior commissure line and acquired axially with a $\mathrm{T} 2 *$-weighted single shot echo planar imaging (EPI) sequence $\left(\mathrm{TR}=1500 \mathrm{~ms}\right.$; $\mathrm{TE}=25 \mathrm{~ms} ; 90^{\circ} \mathrm{RF}$ pulse; acquisition matrix $=64 \times 64 ; \mathrm{FOV}=220 \times 220 \mathrm{~mm}$; in-plane resolution $=220 / 64 \mathrm{~mm}$; slice thickness $=4 \mathrm{~mm} ; 30$ interleaved axial slices). Four dummy scans were collected at the outset of the run and discarded. The EPI sequence consisted of 486 volumes covering the cortical surface and a portion of the cerebellum with a total acquisition time of $12 \mathrm{~min}$ and $24 \mathrm{~s}$. Magnitude and phase images were also acquired (1 min and $40 \mathrm{~s}$ each) for fieldmap-based unwarping $(\mathrm{TR}=700 \mathrm{~ms}$; $\mathrm{TE}=$ 5.0/7.2 ms; FOV $=220 \times 220 \mathrm{~mm}$ matrix; flip angle $=30^{\circ} ;$ slice thickness $=2 \mathrm{~mm}$; 60 interleaved slices).

\section{Data analyses}

Functional neuroimaging data were processed and analyzed using Statistical Parametric Mapping (SPM12, Wellcome Department of Cognitive Neurology, London, UK). The dcm2nii conversion tool was used to convert data from GE DICOM to NIFTI format (Rorden, 2007). The preprocessing pipeline included slice time correction to address nonsequential, interleaved acquisition and realignment of functional images to the first volume of the functional run to adjust for head movement. To account for phase and magnitude variations across the scan, fieldmaps were used to realign and unwarp images. Co-registration of anatomical scans to the first image of the functional scan was followed by registration of anatomical and functional images to the Montreal Neurological Institute (MNI) template. The anatomical image was segmented into bone, air, cerebrospinal fluid, non-brain soft tissue, and brain tissue (i.e., white and gray matter). Deformation fields were applied to permit spatial normalization to MNI space and images were smoothed with a $6.75 \mathrm{~mm}$ full-width half-maximum Gaussian filter.
Following the above pre-processing steps, the General Linear Model (SPM12) was applied to create BOLD signal activation maps of encoding minus control trials and recall minus control trials. A $p<.05$, family-wise-error (FWE) corrected statistical threshold with a minimum of eight contiguous voxels was selected for analyses given optimal balance between Type I and II errors (Lazar, 2008).

ROIs were defined using the WFU PickAtlas version 3.0.5 (Maldjian, Laurienti, Burdette, \& Kraft, 2003; Maldjian, Laurienti, \& Burdette, 2004). Consistent with prior studies (e.g., Bookheimer et al., 2000, 2013; Cabeza \& Nyberg, 2000; Clément \& Belleville, 2009), ROIs during learning trials included medial temporal lobe, supramarginal and angular gyri, precuneus, dorsolateral and ventrolateral prefrontal cortex, anterior and posterior cingulate gyrus, Broca's area, cerebellum, and premotor areas. Recall trials consisted of these ROIs as well as anterior prefrontal cortex, retrosplenial cortex, and cuneus (Cabeza \& Nyberg, 2000).

To assess the anticipated effect of $\mathrm{L}$ and $\mathrm{Z}$ supplementation on brain activity, a $2 \times 2$ flexible factorial model was used in second level ROI analyses with group (placebo vs. supplement) as a between-subjects factor and time (baseline vs. post-intervention) as a within-subjects factor. Significant group $\times$ time interactions within the aforementioned ROIs would be consistent with the expectation that $\mathrm{L}$ and $\mathrm{Z}$ intake alters neural activation relative to placebo. Repeated measure within-group comparisons were used to more specifically characterize significant interactions within ROIs in terms of directionality (i.e., increases $v s$. decreases in BOLD signal). All second level ROI analyses were conducted separately for encoding and retrieval given non-identical neural underpinnings of these two processes (Cabeza \& Nyberg, 2000).

Behavioral performance was also assessed to help verify task engagement and evaluate for intervention effects. Within-scanner self-reported recall of the second word in each pair on the last two recall blocks (maximum score $=10$ ), when learning is expected to be maximal, was calculated. These values were then correlated with actual cued recall assessed immediately post scan to evaluate performance validity. Behavioral performance was also subject to a 
Table 2. Descriptive statistics

\begin{tabular}{lcccc}
\hline \hline & $\begin{array}{c}\text { Placebo group } \\
(n=14)\end{array}$ & $\begin{array}{c}\text { Supplement group } \\
(n=30)\end{array}$ & $\begin{array}{c}p \text {-Value } \\
\left(t \text {-test or } \chi^{2}\right)\end{array}$ & $\begin{array}{c}\text { Effect size } \\
(\text { Cohen's } d)\end{array}$ \\
\hline Age [M (SD)] & $70.43(5.43)$ & $72.43(6.48)$ & .321 & 0.33 \\
Race (\% Caucasian) & $100 \%$ & $100 \%$ & - & - \\
Sex $\%$ female) & $71.43 \%$ & $53.33 \%$ & .419 & 0.25 \\
Education [M(SD)] & $16.71(3.02)$ & $16.73(3.02)$ & .724 & 0.12 \\
WTAR [M(SD)] & $115.29(7.44)$ & $114.43(7.79)$ & .734 & 0.11 \\
GDS [M (SD)] & $1.71(1.89)$ & $2.43(3.09)$ & .427 & 0.27 \\
Dietary intake [M (SD)] & & & .505 & 0.22 \\
$\quad$ Vegetable & $14.25(6.51)$ & $12.97(5.55)$ & .320 & 0.33 \\
Fruit & $10.38(4.88)$ & $12.59(7.50)$ & .772 & .983 \\
Fish & $1.79(1.25)$ & $1.67(1.27)$ & .983 & 0.10 \\
Meat & $4.21(2.12)$ & $4.20(1.95)$ & & 0.01 \\
\hline \hline
\end{tabular}

Note. GDS = Geriatric Depression Scale; $M=$ mean; SD = standard deviation; WTAR = full-scale intelligence quotient predicted from the Wechsler Test of Adult Reading. Dietary intake represents self-reported servings of vegetables, fruits, fish, and meats per week.

$2 \times 2$ mixed design analysis of variance (ANOVA) to investigate the expected group (placebo vs. supplement) by time (baseline vs. post-intervention) interaction.

\section{RESULTS}

\section{Descriptive Statistics}

Sample characteristics and (self-reported) dietary intake of vegetables, fruit, fish, and meat at baseline for both the placebo and supplement groups are presented in Table 2 . Although there were no statistically significant differences between groups, the supplement group was somewhat older than the placebo group (small-to-medium effect size).

Analysis of pill count data and results from bi-monthly compliance phone calls revealed no significant differences between groups with respect to adherence ( $p$ s all $>.05$ ). From the 24 total phone contacts, the average number of self-reported missed pills over the course of the study was only three for both the supplement and control groups. Taken together with the results from MPOD measurements (see below), the pill counts and phone contacts suggest acceptable adherence to the prescribed supplementation regimen.

There were no significant differences between older adults who dropped out of the study $(N=16)$ and those who completed the study $(N=44)$ with respect to age, education level, premorbid intellectual functioning, sex, race, and dietary intake $(p s>.05)$. In addition, MPOD and cognitive performance of individuals who completed the baseline vision $(N=11)$ and fMRI $(N=9)$ sessions but subsequently dropped were comparable to completers ( $p$ s $>.05)$.

\section{MPOD}

Table 3 presents MPOD values, which were used to verify a biological response to $\mathrm{L}$ and $\mathrm{Z}$ supplementation. Given that MPOD data were available at both 8 months and 12 months, we employed an average of these two time points to estimate $\mathrm{L}$ and $\mathrm{Z}$ changes over the course of the intervention. An average was employed to increase the stability of the measurement by doubling the number of observations, particularly given the relatively small number of individuals in the control group $(n=14)$. Importantly, neither the supplement group nor the control group showed a significant change in MPOD between the 8 and 12 month time points. As expected, the supplement group evidenced a significant increase in MPOD across time $[t(29)=2.46 ; p=.016]$ while the placebo group's MPOD remained constant $[t(13)=.05$; $p=.961]$. The supplement group's MPOD was significantly greater than the control group's MPOD following the intervention $[t(42)=2.44 ; p=.019]$, despite the two groups showing comparable MPOD at baseline $(p>.05)$.

We note that the same pattern of results is obtained if the data are analyzed without averaging across 8 and 12 month

Table 3. Intervention effects on MPOD and cognition

\begin{tabular}{llcccc}
\hline \hline & Variable & Baseline & Post-intervention & $p$-Value $(t$-test) & Effect size $($ Cohen's $d)$ \\
\hline Placebo & MPOD [M (SD)] & $0.44(0.14)$ & $0.44(0.19)^{*}$ & .961 & 0.03 \\
$(n=14)$ & Word recall [M (SD)] & $9.36(0.75)$ & $8.21(2.29)$ & .084 & 1.04 \\
Supplement & MPOD [M (SD)] & $0.54(0.19)$ & $0.61(0.22)^{*}$ & .016 & 0.95 \\
$(n=30)$ & Word recall [M (SD)] & $8.87(1.50)$ & $8.80(2.16)$ & .856 & 0.07 \\
\hline \hline
\end{tabular}

Note. $M=$ mean; MPOD = macular pigment optical density; $\mathrm{SD}=$ standard deviation; Word recall = number of words recalled on the final two blocks of the fMRI-adapted verbal learning paradigm (maximum $=10$ ); * = average of 8-month and 12-month (post-intervention) time points. 
Table 4. Zero-order bivariate correlations between MPOD and sample characteristics

\begin{tabular}{lcccrr}
\hline \hline & 1 & 2 & 3 & 4 & 5 \\
\hline 1. Baseline MPOD & - & $0.12(.423)$ & $0.12(.448)$ & $0.10(.509)$ & $-0.02(.900)$ \\
2. Age & & - & $-0.05(.741)$ & $-0.18(.246)$ & $-0.04(.797)$ \\
3. Education & & - & $0.61(<.001)$ & $0.08(.602)$ \\
4. WTAR & & & - & $-.12(.429)$ \\
5. GDS & & & & - \\
\hline \hline
\end{tabular}

Note . GDS $=$ Geriatric Depression Scale; MPOD $=$ macular pigment optical density; WTAR $=$ full-scale intelligence quotient predicted from the Wechsler Test of Adult Reading. Values are presented as Pearson's $r$ ( $p$ value).

time points. That is, the supplement group's MPOD at 12 months showed a significant increase relative to baseline $(p=.03 ; d=0.84)$ while the placebo group's MPOD at 12 months does not significantly differ from baseline.

Zero-order bivariate correlations between MPOD and demographic variables are provided in Table 4. MPOD values at baseline were not significantly related to age, education, depressive symptomatology, or estimated intellectual functioning ( $p$ s all $>.05)$.

\section{Whole-Brain Analyses}

Whole-brain analysis of the encoding minus control contrast in the pooled sample at baseline, independent of supplement status, is shown in Figure 2. Widespread activation was observed in brain regions typically associated with verbal learning in the literature, such as prefrontal (e.g., Broca's area), medial-temporal (e.g., hippocampus), and cerebellar regions with a general tendency for leftlateralization $(p<.05$, FWE-corrected, minimum eight contiguous voxels). Whole-brain analysis of the retrieval minus control contrast at baseline also revealed diffuse activation in brain areas commonly associated with verbal retrieval (see Figure 2), including prefrontal, medial-temporal, parieto-occipital, and cerebellar areas $(p<.05$, FWE-corrected, minimum eight contiguous voxels). Importantly, there were no significant differences in brain activation between supplement and control groups during either learning or retrieval trials at baseline. This finding remained with and without age as a covariate in the between group contrast.

\section{Effects of $L$ and $Z$ on fMRI Performance}

\section{Behavioral}

The number of self-reported successful retrievals during the verbal learning task is presented by group and time point in Table 3. Importantly, the supplement and control groups showed comparable verbal learning performance at baseline $(p>.05)$. The overall sample's average recall was 9.02 out

(a)

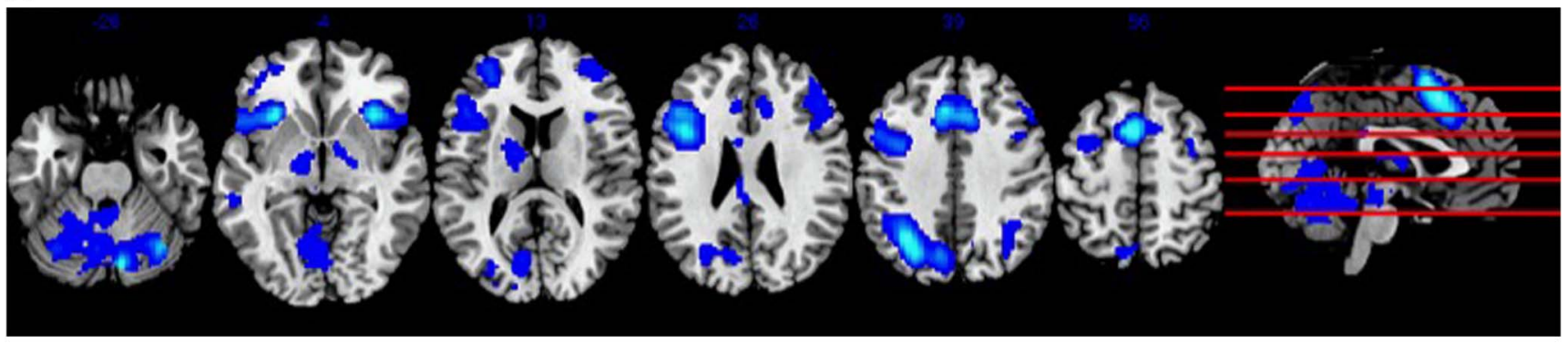

(b)

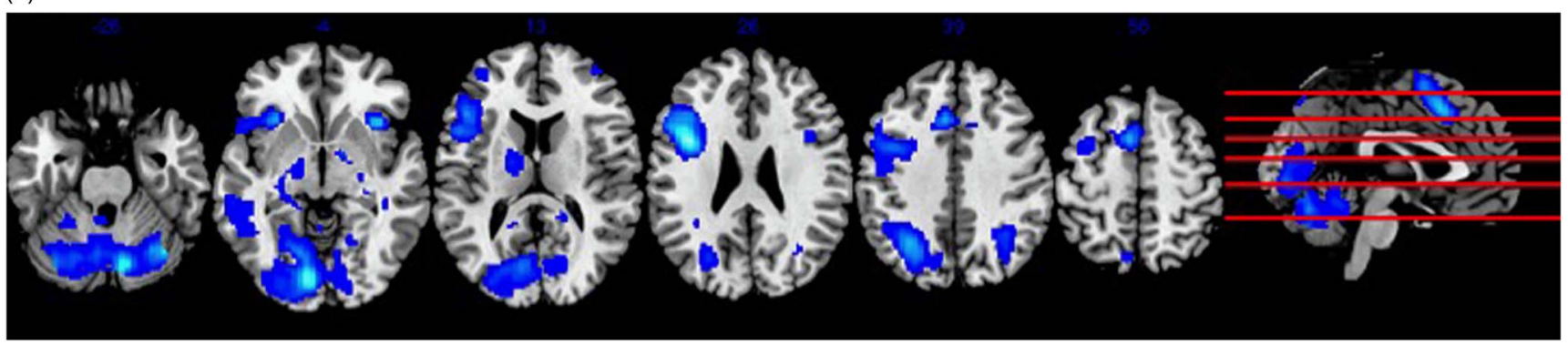

Fig. 2. Panel (a) shows results from whole-brain analysis of the encoding minus control contrast at baseline in the pooled sample, independent of supplement status. Panel (b) similarly depicts whole-brain analysis of the recall minus control contrast in the pooled sample at baseline. Both (a) and (b) represent activity in MNI space superimposed on an anatomical template provided by MRIcron (http://people. cas.sc.edu/rorden/mricron/index.html). 
(a)

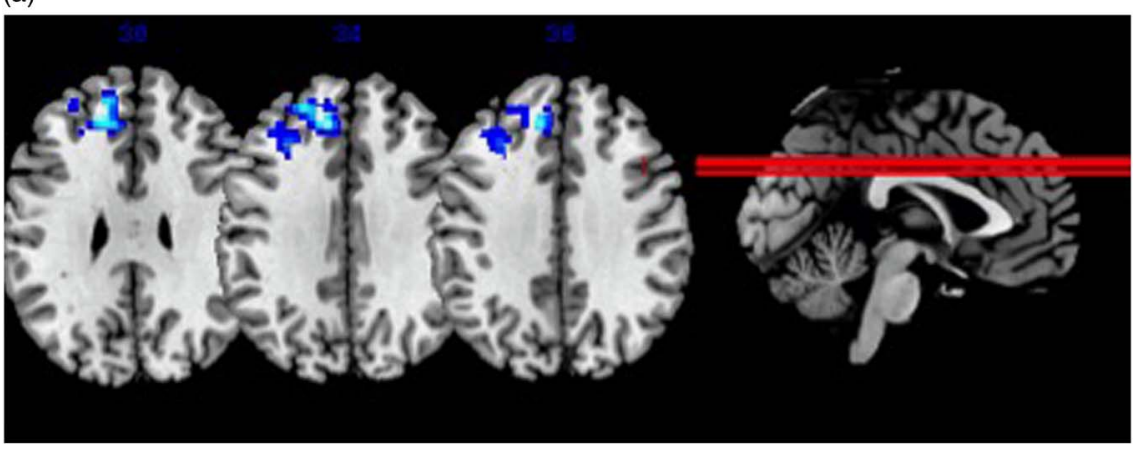

(b)

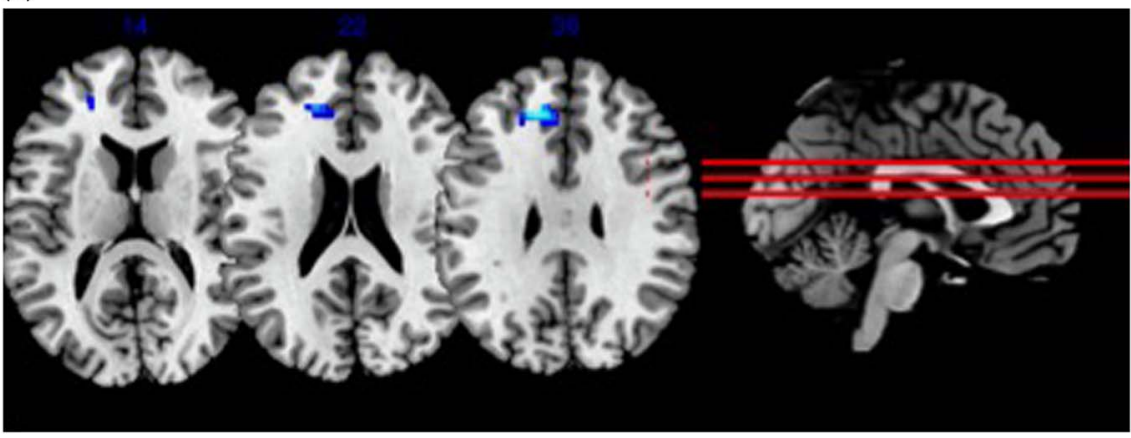

Fig. 3. The above figure depicts significant $(p<.05$, FWE-corrected) group (supplement vs. placebo) $\times$ time (baseline vs. postintervention) interactions during verbal learning in left dorsolateral prefrontal cortex (a) and anterior cingulate cortex (b). Activity is in MNI space and superimposed on an anatomical template provided by MRIcron (http://people.cas.sc.edu/rorden/mricron/install.html).

of the 10 total word pairs at baseline and 8.61 out of 10 postintervention. As expected, within-scanner recall significantly correlated with actual cued recall assessed immediately post scan at both time points (baseline $r=.47 ; p=.001$; postintervention $r=.42 ; p=.005$ ), differing from one another by less than two words on average at baseline (mean difference $=1.61$ words) and post-intervention (mean difference $=$ 0.75 words). The observed congruence suggests that participants were actively engaged in the fMRI task and put forth reasonable effort.

A mixed design ANOVA did not yield a statistically significant group (supplement $v s$. placebo) $\times$ time (baseline $v s$. post-intervention) interaction for number of words recalled within the scanner $[F(1,42)=2.53 ; p=.119]$, although the effect size was large $(d=.84)$. Analysis of simple effects indicated that the supplement group maintained a similar level of performance at post-intervention relative to baseline $[t(29)=-.18 ; p=.856 ; d=.07]$ while the placebo group showed a statistical trend toward decline $[t(13)=-1.87$; $p=.084]$ characterized by a large effect size $(d=1.04)$.

\section{ROI analysis}

Following the encoding minus control contrast, significant group (supplement vs. placebo) $\times$ time (baseline $v s$. postintervention) interactions were observed in left dorsolateral prefrontal cortex $(F=21.34 ; p=.034$, FWE-corrected, cluster size: 498 voxels) and anterior cingulate cortex $(F=19.63 ; p=.028$, FWE-corrected, cluster size: 146 voxels; see Figure 3). Suprathreshold activation was not found in any of the other ROIs, although the effect was approaching significance in right hippocampus $(F=15.13$; $p=.054$, FWE-corrected, cluster size: 20 voxels). To characterize significant interactions, paired $t$-tests revealed increases in BOLD signal in the supplement group from baseline to post-intervention in left dorsolateral prefrontal cortex ( $p=.014 ;$ FWE-corrected) and anterior cingulate cortex ( $p=.016$; FWE-corrected). Importantly, the placebo group did not show activation changes in these regions over the course of the study.

With respect to possible intervention effects during retrieval, a $2 \times 2$ flexible factorial model did not return significant interactions in hypothesized ROIs ( $p$ s all $>.05$, FWEcorrected, minimum 8 contiguous voxels). Exploratory paired $t$-tests did, however, yield significant increases in left dorsolateral prefrontal cortex $(t=4.42 ; p=.045$, FWEcorrected, cluster size: 156 voxels) and anterior cingulate cortex $(t=4.45 ; p=.021$, FWE-corrected, cluster size: 182 voxels) at post-intervention relative to baseline in the supplement group only (see Figure 4), consistent with findings during encoding. No other ROIs showed changes in BOLD signal that survived the FWE correction in exploratory analyses.

\section{DISCUSSION}

$\mathrm{L}$ and $\mathrm{Z}$ supplementation significantly influenced brain function on a verbal learning task relative to placebo. Rather than 
(a)

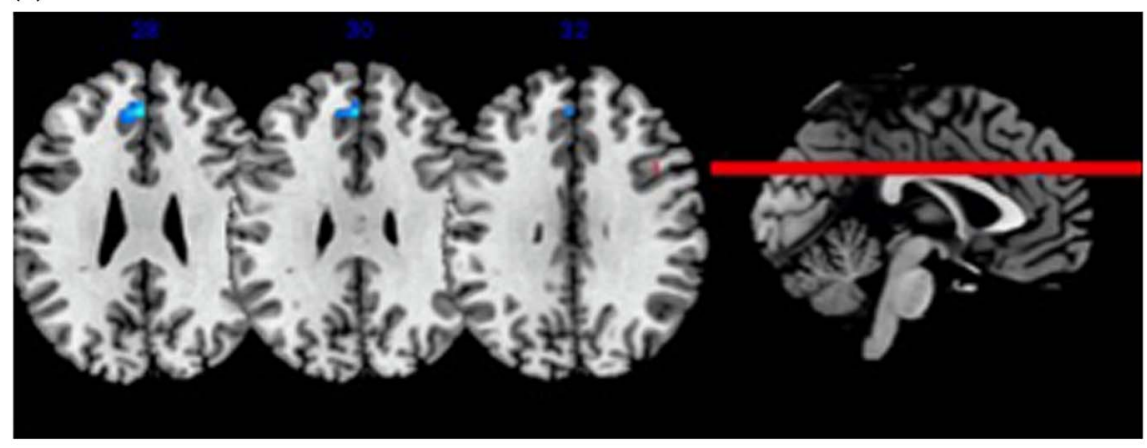

(b)

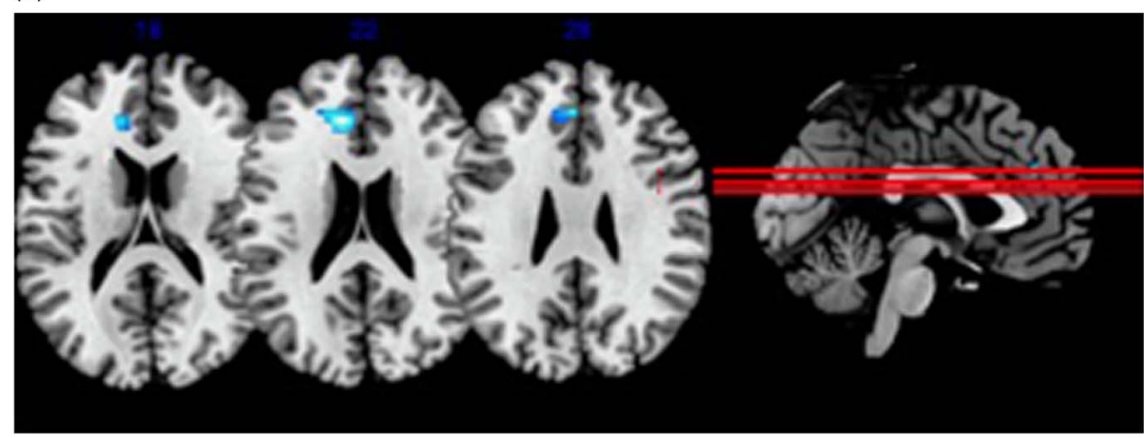

Fig. 4. The above figure shows results from exploratory paired $t$-tests during verbal recall. Significant $(p<.05$, FWE-corrected) increases in left dorsolateral prefrontal cortex (a) and anterior cingulate cortex (b) were observed in the supplement group at post-intervention relative to baseline. The control group did not show activation changes in these regions over the course of the study. Activity is in MNI space and superimposed on an anatomical template provided by MRIcron (http://people.cas.sc.edu/rorden/mricron/install.html).

increasing neural efficiency as predicted, however, carotenoid consumption enhanced BOLD signal in select ROIs, including left dorsolateral prefrontal cortex and anterior cingulate cortex. A similar pattern was observed during verbal retrieval in exploratory within-group analyses but given non-significance in the omnibus interaction, these findings must be interpreted cautiously. It is noteworthy that a nearly significant effect was observed in right hippocampus during learning, which suggests a possible influence of $\mathrm{L}$ and $\mathrm{Z}$ in this region as well. The hippocampal effect was in the hypothesized direction of reduced neural activity, although conclusions are limited without having survived statistical correction for multiple comparisons.

The observed increase in prefrontal BOLD signal seems inconsistent with cross-sectional findings from this sample at baseline, which showed a negative relationship of endogenous $\mathrm{L}$ and $\mathrm{Z}$ levels to neural activity (Lindbergh et al., 2016). However, it is possible the baseline and longitudinal findings are actually complementary. Within the context of the STAC-r (Reuter-Lorenz \& Park, 2014), high levels of L and Z consumption over the course of a lifetime may in fact buffer agerelated neurophysiological decline and reduce the resulting need for compensatory scaffolding. This would be analogous to a "brain maintenance" effect as described by Nyberg, Lövdén, Riklund, Lindenberger, and Bäckman (2012).

Although Lindbergh et al. (2016) measured L and Z status at a single point in time, we speculate that this cross-sectional measurement at least to some extent reflects, and would correlate with, lifetime eating behaviors. This is consistent with empirical evidence suggesting that dietary patterns are relatively stable across time, at least from childhood through adulthood (e.g., Mikkilä, Räsänen, Raitakari, Pietinen, \& Viikari, 2005). MPOD, one of the predictors used by Lindbergh et al. (2016), has demonstrated considerable stability in adults over multiple year spans, particularly relative to serum $\mathrm{L}$ and $\mathrm{Z}$ concentrations (Beatty, Nolan, Kavanagh, \& O'Donovan, 2004; Nolan et al., 2006). Accordingly, to the extent that cross-sectional L and $\mathrm{Z}$ levels (particularly MPOD) approximate lifetime eating behaviors, the Lindbergh et al. (2016) findings may reflect a lifespan neural efficiency effect. Of course, our interpretation relies on the assumption that a "snapshot" measurement of $\mathrm{L}$ and $\mathrm{Z}$ status in old age meaningfully proxies $\mathrm{L}$ and $\mathrm{Z}$ intake across the lifespan, and some studies suggest that MPOD (and presumably brain levels) can change in the event that individuals significantly alter carotenoid intakes (e.g., Hammond et al., 1997; Stringham \& Hammond, 2008). Longitudinal research will be necessary to verify our interpretations, ideally focusing on changes in both MPOD and serum L and $\mathrm{Z}$ concentrations in relation to brain function. Such research may need to begin as early as childhood in light of recent findings that $\mathrm{L}$ and $\mathrm{Z}$ status in 7- to 10 -year-olds positively predicts hippocampal-dependent memory performance (Hassevoort et al., 2017).

Our current RCT findings neither support nor contradict the possibility of a lifespan neural efficiency effect but rather address a somewhat different question involving the neural 
effect of elevated $\mathrm{L}$ and $\mathrm{Z}$ intake circumscribed to a one year period in late life. Results suggest that $\mathrm{L}$ and $\mathrm{Z}$ consumed in this fashion increases cerebral perfusion and enhances neural response during cognitive performance. While further research is required to identify mechanisms underlying this effect, studies using animal models show that antioxidants restore cerebral blood flow following traumatic brain injury (Bitner et al., 2012) and hypoxia-induced metabolic stress (Huang et al., 2013). Aging similarly increases risk for cerebral hypoperfusion, which in turn is associated with cognitive impairment and dementia (D'Esposito, Deouell, \& Gazzaley, 2003; Ruitenberg et al., 2005). L and Z may counter these effects by increasing blood flow to regions at risk for poor cerebral perfusion and age-related deterioration.

The pattern of increased BOLD signal is consistent with a small number of RCTs that have examined fMRI changes related to other dietary factors. As examples, nitrates (Presley et al., 2011), pomegranate juice (Bookheimer et al., 2013), flavanols (Brickman et al., 2014), and fish oil (Boespflug, McNamara, Eliassen, Schidler, \& Krikorian, 2016) have all been found to enhance BOLD signal relative to control conditions. Cholinesterase inhibitors have similarly increased BOLD signal in older adults during memory performance (Goekoop et al., 2004; Saykin et al., 2004). Of note, several of these interventions observed BOLD signal increases in dorsolateral prefrontal cortex and anterior cingulate cortex specifically (e.g., Goekoop et al., 2004; Presley et al., 2011; Saykin et al., 2004), as was found in the present RCT.

Anterior cingulate cortex and dorsolateral prefrontal cortex comprise a closely related functional network (e.g., Fleck, Daselaar, Dobbins, \& Cabeza, 2006; MacDonald, Cohen, Stenger, \& Carter, 2000) and together mediate executive functions (Gasquoine, 2013). Left dorsolateral prefrontal cortex monitors and manipulates verbal information to support encoding (Barbey, Koenigs, \& Grafman, 2013), and more generally subserves cognitive control processes specific to long-term memory formation (MacDonald et al., 2000; Niendam et al., 2012). This is particularly true when relationships must be built between items (e.g., word pairs; Blumenfeld, Parks, Yonelinas, \& Ranganath, 2011) and dorsolateral prefrontal cortex activity positively correlates with success on relational encoding tasks (Blumenfeld \& Ranganath, 2006).

Anterior cingulate cortex is reliably activated on memory tasks as well, particularly when they are challenging or require a high level of attention (Cabeza \& Nyberg, 2000; Niendam et al., 2012; Shenhav, Botvinick, \& Cohen, 2013; Weissman, Gopalakrishnan, Hazlett, \& Woldorff, 2005). Conflict detection and resolution are also rooted in anterior cingulate cortex, which may account for its role in memory decision making (Fleck et al., 2006).

Although $\mathrm{L}$ and Z's behavioral effects on the verbal learning task were not statistically significant, it is notable that the effect size was large. The lack of significance may relate to inadequate power, which is a common phenomenon in fMRI studies that consider behavioral measures (Wilkinson \& Halligan, 2004). A post hoc power analysis revealed that, even with the large effect size we observed, a sample size of 46 (experimental $n=31$; control $n=15$ ) would have been required to detect a significant effect, if present, with a power $(1-\beta)$ of 0.90 (Faul, Erdfelder, Buchner, \& Lang, 2009; Faul, Erdfelder, Lang, \& Buchner, 2007). Given that our sample size following attrition was slightly smaller than this $(N=44)$, conclusions must be limited.

However, our findings tentatively suggest that carotenoid supplementation in old age helps maintain cognitive performance across time, possibly buffering against decline; enhanced cerebral perfusion in prefrontal regions offers a potential neural mechanism for this effect. Alternatively, $\mathrm{L}$ and $\mathrm{Z}$ may not be influencing learning and memory per se, but rather associated cognitive processes (e.g., attention). Finally, the possibility that the effects of $\mathrm{L}$ and $\mathrm{Z}$ supplementation on overt cognitive function in old age are actually quite modest must also be acknowledged. A longer supplementation period may be required (i.e., $>1$ year) or perhaps $\mathrm{L}$ and $\mathrm{Z}$ intake must be elevated across the lifespan to meaningfully influence cognition.

The present study has limitations. Our sample was racially homogenous, high functioning, and well educated. Future research is warranted to determine whether results generalize to populations characterized by greater diversity and cognitive variability. This is particularly important given that elevated socioeconomic position may reduce the strength of the relationship between dietary factors and cognition (Akbaraly, Singh-Manoux, Marmot, \& Brunner, 2009). In light of the high average number of words recalled on the verbal learning task, future RCTs should consider changes using more sensitive neuropsychological measures and in other cognitive domains. The generalizability of our findings may also be influenced by the relatively high number of individuals who dropped out of the study ( 27\%), although it is encouraging that non-completers did not display any significant differences from completers on demographic and dietary factors. Still, it must be kept in mind that our dietary assessment provided only limited information about food categories, and it is possible differences existed on more specific nutritional variables that have been shown to relate to cognition (e.g., omega-3 fatty acids; Mazereeuw, Lanctôt, Chau, Swardfager, \& Hermann, 2012). Finally, as with most nutrition studies, there was no true "control" group; $\mathrm{L}$ and $\mathrm{Z}$ are abundant in a variety of foods. In many respects, the observation of any brain effect beyond routine $\mathrm{L}$ and $\mathrm{Z}$ consumption in an intact and presumably well-nourished sample speaks to the robust potential for carotenoids to influence neurocognitive function.

Despite its limitations, this is the first RCT to investigate the effects of $\mathrm{L}$ and $\mathrm{Z}$ supplementation on neural function in vivo during cognitive task performance. More broadly, our results add to the paucity of research that has evaluated the potential of nutrition, a modifiable and inexpensive lifestyle factor, to promote brain health.

\section{ACKNOWLEDGMENTS}

This research project was funded in part by Abbott Nutritional Products (Columbus, OH; research grant to B.R.H., L.M.R., L.S.M.) and the University of Georgia's Bio-Imaging Research Center 
(administrative support, L.S.M.). DSM Nutritional Products (Switzerland) provided the supplements and placebos. Additionally, L.M.R. was an employee of Abbott Nutrition during a portion of the grant period while holding a joint appointment at the University of Georgia. B.R.H. has consulted for Abbott Nutrition. No other potential conflicts of interest exist for any of the study authors, including A.N.P., C.A.L., C.M.M., and D.P.T. All statistical analyses were completed independently of supporting agencies.

\section{REFERENCES}

Akbaraly, N.T., Faure, H., Gourlet, V., Favier, A., \& Berr, C. (2007). Plasma carotenoid levels and cognitive performance in an elderly population: Results of the EVA Study. The Journals of Gerontology Series A: Biological Sciences and Medical Sciences, 62(3), 308-316.

Akbaraly, T.N., Singh-Manoux, A., Marmot, M.G., \& Brunner, E.J. (2009). Education attenuates the association between dietary patterns and cognition. Dementia and Geriatric Cognitive Disorders, 27(2), 147-154.

Barbey, A.K., Koenigs, M., \& Grafman, J. (2013). Dorsolateral prefrontal contributions to human working memory. Cortex, 49(5), 1195-1205.

Beatty, S., Nolan, J., Kavanagh, H., \& O’Donovan, O. (2004). Macular pigment optical density and its relationship with serum and dietary levels of lutein and zeaxanthin. Archives of Biochemistry and Biophysics, 430(1), 70-76. http://doi.org/ 10.1016/j.abb.2004.03.015

Bernstein, P.S., Khachik, F., Carvalho, L.S., Muir, G.J., Zhao, D.Y., \& Katz, N.B. (2001). Identification and quantitation of carotenoids and their metabolites in the tissues of the human eye. Experimental Eye Research, 72(3), 215-223.

Bertram, J.S. (1999). Carotenoids and gene regulation. Nutrition Reviews, 57(6), 182-191. http://doi.org/10.1111/j.1753-4887. 1999.tb06941.x

Bitner, B.R., Marcano, D.C., Berlin, J.M., Fabian, R.H., Cherian, L., Culver, J.C., ... Tour, J.M. (2012). Antioxidant carbon particles improve cerebrovascular dysfunction following traumatic brain injury. ACS Nano, 6(9), 8007-8014.

Blumenfeld, R.S., Parks, C.M., Yonelinas, A.P., \& Ranganath, C. (2011). Putting the pieces together: The role of dorsolateral prefrontal cortex in relational memory encoding. Journal of Cognitive Neuroscience, 23(1), 257-265.

Blumenfeld, R.S., \& Ranganath, C. (2006). Dorsolateral prefrontal cortex promotes long-term memory formation through its role in working memory organization. The Journal of Neuroscience, 26(3), 916-925.

Boespflug, E.L., McNamara, R.K., Eliassen, J.C., Schidler, M.D., \& Krikorian, R. (2016). Fish oil supplementation increases eventrelated posterior cingulate activation in older adults with subjective memory impairment. The Journal of Nutrition, Health and Aging, 20(2), 161-169. http://doi.org/10.1007/s12603-015-0609-6

Bokov, A., Chaudhuri, A., \& Richardson, A. (2004). The role of oxidative damage and stress in aging. Mechanisms of Ageing and Development, 125(10-11), 811-826. http://doi.org/10.1016/j. mad.2004.07.009

Bone, R.A., Landrum, J.T., \& Tarsis, S.L. (1985). Preliminary identification of the human macular pigment. Vision Research, 25(11), 1531-1535.

Bookheimer, S.Y., Renner, B.A., Ekstrom, A., Li, Z., Henning, S.M., Brown, J.A., ... Small, G.W. (2013). Pomegranate juice augments memory and fMRI activity in middle-aged and older adults with mild memory complaints. Evidence-Based Complementary and Alternative Medicine. http://doi.org/10.1155/2013/946298

Bookheimer, S.Y., Strojwas, M.H., Cohen, M.S., Saunders, A.M., Pericak-Vance, M.A., Mazziotta, J.C., \& Small, G.W. (2000). Patterns of brain activation in people at risk for Alzheimer's disease. The New England Journal of Medicine, 343(7), 450-456. http://doi.org/10.1056/NEJM200008173430701

Braskie, M.N., Small, G.W., \& Bookheimer, S.Y. (2009). Entorhinal cortex structure and functional MRI response during an associative verbal memory task. Human Brain Mapping, 30(12), 3981-3992. http://doi.org/10.1002/hbm.20823

Brickman, A.M., Khan, U.A., Provenzano, F.A., Yeung, L.K., Suzuki, W., Schroeter, H., ... Small, S.A. (2014). Enhancing dentate gyrus function with dietary flavanols improves cognition in older adults. Nature Neuroscience, 17(12), 1798-1803. http:// doi.org/10.1038/nn.3850

Britton, G., Liaaen-Jensen, S., \& Pfander, H. (2004). Carotenoids: Handbook. Basel, Switzerland: Springer.

Butterfield, D.A., Bader Lange, M.L., \& Sultana, R. (2010). Involvements of the lipid peroxidation product, $\mathrm{HNE}$, in the pathogenesis and progression of Alzheimer's disease. Biochimica et Biophysica Acta, 1801(8), 924-929. http://doi.org/10.1016/j. bbalip.2010.02.005

Cabeza, R., \& Nyberg, L. (2000). Imaging Cognition II: An empirical review of 275 PET and fMRI studies. Journal of Cognitive Neuroscience, 12(1), 1-47. http://doi.org/10.1162/ 08989290051137585

Clément, F., \& Belleville, S. (2009). Test-retest reliability of fMRI verbal episodic memory paradigms in healthy older adults and in persons with mild cognitive impairment. Human Brain Mapping, 30(12), 4033-4047. http://doi.org/10.1002/ hbm.20827

Craft, N.E., Haitema, T.B., Garnett, K.M., Fitch, K.A., \& Dorey, C.K. (2004). Carotenoid, tocopherol, and retinol concentrations in elderly human brain. The Journal of Nutrition, Health and Aging, 8(3), 156-162.

D'Esposito, M., Deouell, L.Y., \& Gazzaley, A. (2003). Alterations in the BOLD fMRI signal with ageing and disease: A challenge for neuroimaging. Nature Reviews Neuroscience, 4(11), 863-872.

Erdman, J.W., Smith, J.W., Kuchan, M.J., Mohn, E.S., Johnson, E.J., Rubakhin, S.S., ... Neuringer, M. (2015). Lutein and brain function. Foods, 4(4), 547-564. http://doi.org/10.3390/foods4040547

Faul, F., Erdfelder, E., Buchner, A., \& Lang, A.-G. (2009). Statistical power analyses using G*Power 3.1: Tests for correlation and regression analyses. Behavior Research Methods, 41, 1149-1160.

Faul, F., Erdfelder, E., Lang, A.-G., \& Buchner, A. (2007). G*Power 3: A flexible statistical power analysis program for the social, behavioral, and biomedical sciences. Behavior Research Methods, 39, 175-191.

Feart, C., Letenneur, L., Helmer, C., Samieri, C., Schalch, W., Etheve, S., ... Barberger-Gateau, P. (2015). Plasma carotenoids are inversely associated with dementia risk in an elderly French cohort. The Journals of Gerontology Series A: Biological Sciences and Medical Sciences, 71(5), 683-688.

Feeney, J., Finucane, C., Savva, G.M., Cronin, H., Beatty, S., Nolan, J.M., \& Kenny, R.A. (2013). Low macular pigment optical density is associated with lower cognitive performance in a large, population-based sample of older adults. Neurobiology of Aging, 34(11), 2449-2456. http://doi.org/10.1016/j.neurobiolaging. 2013.05.007 
Fleck, M.S., Daselaar, S.M., Dobbins, I.G., \& Cabeza, R. (2006). Role of prefrontal and anterior cingulate regions in decisionmaking processes shared by memory and nonmemory tasks. Cerebral Cortex, 16(11), 1623-1630.

Gasquoine, P.G. (2013). Localization of function in anterior cingulate cortex: From psychosurgery to functional neuroimaging. Neuroscience and Biobehavioral Reviews, 37(3), 340-348.

Goekoop, R., Rombouts, S.A., Jonker, C., Hibbel, A., Knol, D.L., Truyen, L., ... Scheltens, P. (2004). Challenging the cholinergic system in mild cognitive impairment: A pharmacological fMRI study. NeuroImage, 23(4), 1450-1459.

Hammond, B.R., Johnson, E.J., Russell, R.M., Krinsky, N.I., Yeum, K.J., Edwards, R.B., \& Snodderly, D.M. (1997). Dietary modification of human macular pigment density. Investigative Ophthalmology and Visual Science, 38(9), 1795-1801.

Hammond, B.R., Wooten, B.R., \& Smollon, B. (2005). Assessment of the validity of in vivo methods of measuring human macular pigment optical density. Optometry and Vision Science, 82(5), 387-404.

Hassevoort, K.M., Khazoum, S.E., Walker, J.A., Barnett, S.M., Raine, L.B., Hammond, B.R., ... Cohen, N.J. (2017). Macular carotenoids, aerobic fitness, and central adiposity are associated differentially with hippocampal-dependent relational memory in preadolescent children. The Journal of Pediatrics. doi: 10.1016/j. jpeds.2017.01.016

Heneka, M.T., Carson, M.J., El Khoury, J., Landreth, G.E., Brosseron, F., Feinstein, D.L., ... Kummer, M.P. (2015). Neuroinflammation in Alzheimer's disease. The Lancet: Neurology, 14(4), 388-405. http://doi.org/10.1016/S1474-4422(15) 70016-5

Huang, S., Du, F., Shih, Y.Y.I., Shen, Q., Gonzalez-Lima, F., \& Duong, T.Q. (2013). Methylene blue potentiates stimulus-evoked fMRI responses and cerebral oxygen consumption during normoxia and hypoxia. NeuroImage, 72, 237-242.

Johnson, E.J. (2012). A possible role for lutein and zeaxanthin in cognitive function in the elderly. The American Journal of Clinical Nutrition, 96(5), 1161-1165.

Johnson, E.J. (2014). Role of lutein and zeaxanthin in visual and cognitive function throughout the lifespan. Nutrition Reviews, 72(9), 605-612. http://doi.org/10.1111/nure.12133

Johnson, E.J., McDonald, K., Caldarella, S.M., Chung, H., Troen, A. M., \& Snodderly, D.M. (2008). Cognitive findings of an exploratory trial of docosahexaenoic acid and lutein supplementation in older women. Nutritional Neuroscience, 11(2), 75-83.

Johnson, E.J., Vishwanathan, R., Johnson, M.A., Hausman, D.B., Davey, A., Scott, T.M., ... Poon, L.W. (2013). Relationship between serum and brain carotenoids, $\alpha$-tocopherol, and retinol concentrations and cognitive performance in the oldest old from the Georgia Centenarian Study. Journal of Aging Research. http://doi.org/10.1155/2013/951786

Kang, J.H., Ascherio, A., \& Grodstein, F. (2005). Fruit and vegetable consumption and cognitive decline in aging women. Annals of Neurology, 57(5), 713-720. http://doi.org/10.1002/ana.20476

Kelly, D., Coen, R.F., Akuffo, K.O., Beatty, S., Dennison, J., Moran, R., ... Nolan, J.M. (2015). Cognitive function and its relationship with macular pigment optical density and serum concentrations of its constituent carotenoids. Journal of Alzheimer's Disease, 48(1), 261-277.

Kesse-Guyot, E., Andreeva, V.A., Ducros, V., Jeandel, C., Julia, C., Hercberg, S., \& Galan, P. (2014). Carotenoid-rich dietary patterns during midlife and subsequent cognitive function. The British Journal of Nutrition, 111(5), 915-923.
Khachik, F., Beecher, G.R., Goli, M.B., \& Lusby, W.R. (1991). Separation, identification, and quantification of carotenoids in fruits, vegetables and human plasma by high performance liquid chromatography. Pure and Applied Chemistry, 63(1), 71-80. http://doi.org/10.1351/pac199163010071

Krinsky, N.I., Mayne, S.T., \& Sies, H. (2004). Carotenoids in Health and Disease. Boca Raton, FL: CRC Press.

Kvansakul, J., Rodriguez-Carmona, M., Edgar, D.F., Barker, F.M., Koëpcke, W., Schalch, W., \& Barbur, J.L. (2006). Supplementation with the carotenoids lutein or zeaxanthin improves human visual performance. Ophthalmic and Physiological Optics, 26(4), 362-371.

Landrum, J.T., Bone, R.A., Joa, H., Kilburn, M.D., Moore, L.L., \& Sprague, K.E. (1997). A one year study of the macular pigment: The effect of 140 days of a lutein supplement. Experimental Eye Research, 65(1), 57-62.

Lazar, N. (2008). The statistical analysis of functional MRI data. New York, NY: Springer.

Lee, Y., Kim, J., \& Back, J.H. (2009). The influence of multiple lifestyle behaviors on cognitive function in older persons living in the community. Preventive Medicine, 48(1), 86-90. http://doi. org/10.1016/j.ypmed.2008.10.021

Lindbergh, C.A., Mewborn, C., Hammond, B.R., Renzi-Hammond, L.M., Curran-Celentano, J.M., \& Miller, L.S. (2016). The relationship of lutein and zeaxanthin levels to neurocognitive functioning: An fMRI study of older adults. Journal of the International Neuropsychological Society, 22, 1-12. doi: $10.1017 / \mathrm{S} 1355617716000850$

Ma, L., Dou, H.-L., Wu, Y.-Q., Huang, Y.-M., Huang, Y.-B., Xu, X.-R., ... Lin, X.-M. (2012). Lutein and zeaxanthin intake and the risk of age-related macular degeneration: A systematic review and meta-analysis. British Journal of Nutrition, 107(3), 350-359. http://doi.org/10.1017/S0007114511004260

Ma, L., Lin, X.M., Zou, Z.Y., Xu, X.R., Li, Y., \& Xu, R. (2009). A 12-week lutein supplementation improves visual function in Chinese people with long-term computer display light exposure. British Journal of Nutrition, 102(2), 186-190.

MacDonald, A.W., Cohen, J.D., Stenger, V.A., \& Carter, C.S. (2000). Dissociating the role of the dorsolateral prefrontal and anterior cingulate cortex in cognitive control. Science, 288(5472), 1835-1838.

Maci, S., Fonseca, B., \& Zhu, Y. (2016). The role of lutein in brain health and function. Nutrafoods, 15, 179-188. doi: 10.17470/nf016-1014-3

Maldjian, J.A., Laurienti, P.J., \& Burdette, J.H. (2004). Precentral gyrus discrepancy in electronic versions of the Talairach Atlas. NeuroImage, 21(1), 450-455.

Maldjian, J.A., Laurienti, P.J., Burdette, J.B., \& Kraft, R.A. (2003). An automated method for neuroanatomic and cytoarchitectonic atlasbased interrogation of fMRI data sets. NeuroImage, 19, 1233-1239.

Malinow, M.R., Feeney-Burns, L., Peterson, L.H., Klein, M.L., \& Neuringer, M. (1980). Diet-related macular anomalies in monkeys. Investigative Ophthalmology and Visual Science, 19(8), 857-863.

Mares, J.A., LaRowe, T.L., Snodderly, D.M., Moeller, S.M., Gruber, M.J., Klein, M.L., ... Chappell, R.J. (2006). Predictors of optical density of lutein and zeaxanthin in retinas of older women in the Carotenoids in Age-Related Eye Disease Study, an ancillary study of the Women's Health Initiative. The American Journal of Clinical Nutrition, 84(5), 1107-1122.

Mazereeuw, G., Lanctôt, K.L., Chau, S.A., Swardfager, W., \& Herrmann, N. (2012). Effects of omega-3 fatty acids on cognitive performance: A meta-analysis. Neurobiology of Aging, 33(7), $17-29$. 
Mikkilä, V., Räsänen, L., Raitakari, O.T., Pietinen, P., \& Viikari, J. (2005). Consistent dietary patterns identified from childhood to adulthood: The Cardiovascular Risk in Young Finns Study. British Journal of Nutrition, 93(6), 923-931.

Min, J., \& Min, K. (2014). Serum lycopene, lutein and zeaxanthin, and the risk of Alzheimer's disease mortality in older adults. Dementia and Geriatric Cognitive Disorders, 37(3-4), 246-256.

Morris, M.C., Evans, D.A., Tangney, C.C., Bienias, J.L., \& Wilson, R.S. (2006). Associations of vegetable and fruit consumption with age-related cognitive change. Neurology, 67(8), 1370-1376. http://doi.org/10.1212/01.wnl.0000240224.38978.d8

Murray, I.J., Makridaki, M., van der Veen, R.L., Carden, D., Parry, N.R., \& Berendschot, T.T. (2013). Lutein supplementation over a one-year period in early AMD might have a mild beneficial effect on visual acuity: The CLEAR Study. Investigative Ophthalmology \& Visual Science, 54(3), 1781-1788.

Niendam, T.A., Laird, A.R., Ray, K.L., Dean, Y.M., Glahn, D.C., \& Carter, C.S. (2012). Meta-analytic evidence for a superordinate cognitive control network subserving diverse executive functions. Cognitive, Affective, and Behavioral Neuroscience, 12(2), 241-268.

Nolan, J.M., Loskutova, E., Howard, A.N., Moran, R., Mulcahy, R., Stack, J., ... Thurnham, D.I. (2014). Macular pigment, visual function, and macular disease among subjects with Alzheimer's disease: An exploratory study. Journal of Alzheimer's Disease, 42(4), 1191-1202.

Nolan, J.M., Stack, J., Mellerio, J., Godhinio, M., O’Donovan, O., Neelam, K., \& Beatty, S. (2006). Monthly consistency of macular pigment optical density and serum concentrations of lutein and zeaxanthin. Current Eye Research, 31(2), 199-213.

Nyberg, L., Lövdén, M., Riklund, K., Lindenberger, U., \& Bäckman, L. (2012). Memory aging and brain maintenance. Trends in Cognitive Sciences, 16(5), 292-305. http://doi.org/ 10.1016/j.tics.2012.04.005

Park, D.C., \& Reuter-Lorenz, P. (2009). The adaptive brain: Aging and neurocognitive scaffolding. Annual Review of Psychology, 60, 173-196. http://doi.org/10.1146/annurev.psych.59.103006.093656

Presley, T.D., Morgan, A.R., Bechtold, E., Clodfelter, W., Dove R.W., Jennings, J.M., ... Miller, G.D. (2011). Acute effect of a high nitrate diet on brain perfusion in older adults. Nitric Oxide: Biology and Chemistry, 24(1), 34-42. http://doi.org/10.1016/j. niox.2010.10.002

Renzi, L.M., Dengler, M.J., Puente, A., Miller, L.S., \& Hammond B.R. (2014). Relationships between macular pigment optical density and cognitive function in unimpaired and mildly cognitively impaired older adults. Neurobiology of Aging, 35(7), 1695-1699. http://doi.org/10.1016/j.neurobiolaging.2013.12.024

Renzi, L.M., \& Hammond, B.R. (2010). The relation between the macular carotenoids, lutein and zeaxanthin, and temporal vision. Ophthalmic and Physiological Optics, 30(4), 351-357. http://doi. org/10.1111/j.1475-1313.2010.00720.x

Renzi, L., Iannaccone, A., Johnson, E., \& Kritchevsky, S. (2008). The relation between serum xanthophylls, fatty acids, macular pigment and cognitive function in the Health ABC Study. FASEB Journal, 22, 877.5. doi: 10.1096/fj.1530-6860

Reuter-Lorenz, P.A., \& Park, D.C. (2014). How does it STAC up? Revisiting the Scaffolding Theory of Aging and Cognition. Neuropsychology Review, 24(3), 355-370. http://doi.org/ 10.1007/s11065-014-9270-9

Rorden, C. (2007). DCM2NII (Computer software).

Rosano, C., Marsland, A.L., \& Gianaros, P.J. (2012). Maintaining brain health by monitoring inflammatory processes: A mechanism to promote successful aging. Aging and Disease, 3(1), 16-33.
Ruitenberg, A., den Heijer, T., Bakker, S.L., van Swieten, J.C., Koudstaal, P.J., Hofman, A., \& Breteler, M. (2005). Cerebral hypoperfusion and clinical onset of dementia: The Rotterdam Study. Annals of Neurology, 57(6), 789-794.

SanGiovanni, J.P., \& Neuringer, M. (2012). The putative role of lutein and zeaxanthin as protective agents against age-related macular degeneration: Promise of molecular genetics for guiding mechanistic and translational research in the field. The American Journal of Clinical Nutrition, 96(5), 1223-1233. doi: http://doi. org/10.3945/ajcn.112.038240

Saykin, A.J., Wishart, H.A., Rabin, L.A., Flashman, L.A., McHugh, T.L., Mamourian, A.C., \& Santulli, R.B. (2004). Cholinergic enhancement of frontal lobe activity in mild cognitive impairment. Brain, 127(7), 1574-1583.

Shenhav, A., Botvinick, M.M., \& Cohen, J.D. (2013). The expected value of control: An integrative theory of anterior cingulate cortex function. Neuron, 79(2), 217-240.

Stahl, W., \& Sies, H. (2001). Effects of carotenoids and retinoids on gap junctional communication. Biofactors, 15(2-4), 95.

Stringham, J.M., \& Hammond, B.R. (2008). Macular pigment and visual performance under glare conditions. Optometry \& Vision Science, 85(2), 82-88.

Stringham, J.M., Hammond, B.R., Nolan, J.M., Wooten, B.R., Mammen, A., Smollon, W., \& Snodderly, D.M. (2008). The utility of using customized heterochromatic flicker photometry (cHFP) to measure macular pigment in patients with age-related macular degeneration. Experimental Eye Research, 87(5), 445-453.

Vishwanathan, R., Iannaccone, A., Scott, T.M., Kritchevsky, S.B., Jennings, B.J., Carboni, G., ... Johnson, E.J. (2014). Macular pigment optical density is related to cognitive function in older people. Age and Ageing, 43(2), 271-275. http://doi.org/ 10.1093/ageing/aft210

Vishwanathan, R., Neuringer, M., Snodderly, D.M., Schalch, W., \& Johnson, E.J. (2013). Macular lutein and zeaxanthin are related to brain lutein and zeaxanthin in primates. Nutritional Neuroscience, 16(1), 21-29. http://doi.org/10.1179/147683051 2Y.0000000024

Vishwanathan, R., Schalch, W., \& Johnson, E.J. (2016). Macular pigment carotenoids in the retina and occipital cortex are related in humans. Nutritional Neuroscience, 19(3), 95-101. http://doi. org/10.1179/1476830514Y.0000000141

Wechsler, D. (2001). Wechsler Test of Adult Reading: WTAR. San Antonio, TX: Psychological Corporation.

Wechsler, D. (2009). The Wechsler Memory Scale-Fourth Edition (WMS-IV). San Antonio, TX: Pearson Assessments.

Weigert, G., Kaya, S., Pemp, B., Sacu, S., Lasta, M., Werkmeister, R.M., ... Schmetterer, L. (2011). Effects of lutein supplementation on macular pigment optical density and visual acuity in patients with age-related macular degeneration. Investigative Ophthalmology \& Visual Science, 52(11), 8174-8178.

Weissman, D.H., Gopalakrishnan, A., Hazlett, C.J., \& Woldorff M.G. (2005). Dorsal anterior cingulate cortex resolves conflict from distracting stimuli by boosting attention toward relevant events. Cerebral Cortex, 15(2), 229-237.

Widomska, J., \& Subczynski, W.K. (2014). Why has nature chosen lutein and zeaxanthin to protect the retina? Journal of Clinical and Experimental Ophthalmology, 5(1). http://doi.org/10.4172/ 2155-9570.1000326

Wilkinson, D., \& Halligan, P. (2004). The relevance of behavioural measures for functional-imaging studies of cognition. Nature Reviews Neuroscience, 5(1), 67-73. 
Yagi, A., Fujimoto, K., Michihiro, K., Goh, B., Tsi, D., \& Nagai, H. (2009). The effect of lutein supplementation on visual fatigue: A psychophysiological analysis. Applied Ergonomics, 40(6), 1047-1054.

Yesavage, J.A., Brink, T.L., Rose, T.L., Lum, O., Huang, V., Adey, M., ... Leirer, V.O. (1983). Development and validation of a geriatric depression screening scale: A preliminary report. Journal of Psychiatric Research, 17(1), 37-49. http://doi.org/ 10.1016/0022-3956(82)90033-4

Zamroziewicz, M.K., \& Barbey, A.K. (2016). Nutritional cognitive neuroscience: Innovations for healthy brain aging. Frontiers in Neuroscience, 10, 1-10. doi: 10.3389/fnins.2016.00240 